\title{
Photodynamic Therapy Delays Cutaneous Wound Healing in Mice
}

\author{
Yan Sun ${ }^{1,2}$, Mamiko Tosa ${ }^{2}$, Hiroya Takada ${ }^{2}$ and Rei Ogawa ${ }^{2}$ \\ ${ }^{1}$ Department of Dermatology, The First Hospital of China Medical University, Shenyang, China \\ ${ }^{2}$ Department of Plastic, Reconstructive and Aesthetic Surgery, Nippon Medical School, Tokyo, Japan
}

\begin{abstract}
Background: Cutaneous wound healing is a complex, dynamic physiological process. Traditional methods of promoting wound healing are not always effective. Consequently, alternative modalities, such as photodynamic therapy (PDT), are needed. We examined the effectiveness and underlying mechanisms of PDT in a murine model of acute wound healing.

Methods: Two excisional wounds were produced, one on each side of the midline, in C57bL/6J mice. Methyl 5-aminolevulinate hydrochloride (MAL) was applied to the right-side wound. After 1 to 3 hours of incubation, the wound was irradiated with red light. The left-side wound was not treated with MAL or red light. On Day 14, the wounds were excised and subjected to histological and immunohistochemical analysis.
\end{abstract}

Results: During the first week, no difference was seen between the two sides. However, at week 2, PDT-treated wounds exhibited delayed re-epithelialization. On Day 14, hematoxylin and eosin (HE) staining showed a continuous epithelial lining in untreated wounds. In contrast, PDT-treated wounds partially lacked epithelium in the wound bed. Masson's Trichrome (MTC) staining showed a thicker dermis and more collagen fibers and inflammatory cells in PDT-treated wounds than in untreated wounds. Immunohistochemical analyses showed significantly fewer CD $31^{+}$blood vessels and greater collagen III density in PDT-treated wounds than in untreated wounds. However, treated and untreated wounds did not differ in collagen I density.

Conclusions: PDT delayed acute wound healing in a murine model of secondary intention wound healing. (J Nippon Med Sch 2020; 87: 110-117)

Key words: photodynamic therapy, wound healing, mice

\section{Introduction}

Cutaneous wound healing is a complex and dynamic physiological process during which damaged tissues are repaired and skin integrity is restored. Hemostasis is followed by three sequential, albeit overlapping, phases: the inflammatory, proliferative, and remodeling phases ${ }^{1,2}$. Many cell types, cytokines, and other mediators are involved in this process, but the mechanism remains incompletely understood ${ }^{3}$.

In some patients, it is difficult to achieve wound healing, and a long recovery period is needed to obtain wound closure. This can be a significant burden on patients and health care systems. A systematic review of studies published between 1996 and 2008 showed that at any given time, $27 \%$ to $50 \%$ of all hospital beds in Europe are occupied by patients with acute or chronic wounds. Moreover, chronic wounds are a particularly important health care problem: in one UK study, more than half of all patients with wounds in all health care settings had chronic wounds. Various factors delay wound healing, including older age, diabetes, smoking, wound size and location ${ }^{5}$, and infection ${ }^{6}$. Various dressings and topical products promote proper wound healing; negative pressure wound therapy ${ }^{7}$ and skin grafts ${ }^{8}$ can also be useful. However, these methods are not always effective'. Consequently, alternative modalities that reliably achieve good cutaneous wound healing are needed.

Correspondence to Yan Sun, Department of Dermatology, The First Hospital of China Medical University, 155 North Nanjing

Street, Shenyang 110001, China

E-mail: sunyan805@163.com

https://doi.org/10.1272/jnms.JNMS.2020_87-301

Journal Website (https://www.nms.ac.jp/sh/jnms/) 
One such possibility is photodynamic therapy (PDT)-a photochemical therapy that involves a photosensitizer, light, and endogenous oxygen. Photosensitizers are precursors of protoporphyrin (Pp) IX, a heterocyclic macrocycle organic molecule that generates reactive oxygen species (ROS) from oxygen when it is excited by light. Thus, when a photosensitizer is applied to a target (eg, microbes and proliferating tumors and other cells), it is taken up and produces ROS when irradiated. The resulting ROS kill the target cells by necrosis, apoptosis, and/ or autophagy ${ }^{10}$. The possibility of PDT first arose in 1841, when Scherer extracted hematoporphyrin from dried blood by removing iron. Later studies showed that treating microbes, erythrocytes, guinea pigs, and humans with hematoporphyrin greatly augmented the damaging effects of light. Thus, hematoporphyrin was the first photosensitizer to be discovered ${ }^{11}$.

PDT was initially used to treat superficial nonmelanoma skin cancers such as basal cell carcinoma ${ }^{12}$, Bowen disease ${ }^{13}$, and actinic keratosis ${ }^{14}$ and was shown to be effective, noninvasive, and safe. Recently, it has been used to treat acne ${ }^{15}$, rosacea ${ }^{16}$, and other skin conditions. There is thus considerable interest in the therapeutic effects of PDT on cutaneous wound healing. Indeed, several recent studies reported that it effectively promotes closure of chronic venous ulcers ${ }^{17}$ and diabetic ulcers ${ }^{18}$ in humans and that it aids in the healing of infected burn wounds by killing microbes ${ }^{19,20}$. However, Mills et al reported that PDT delayed early wound healing of excisional wounds in healthy humans, although it did improve later outcomes such as cosmetic appearance and dermal structure ${ }^{5}$. These inconsistent findings, and the fact that few studies have examined the mechanisms by which PDT affects wound healing, suggest that the role of PDT in wound healing therapeutics warrants further study. We therefore examined the effectiveness and underlying mechanisms of PDT in a murine model of acute wound healing.

\section{Materials and Methods}

Eight-week-old male C57bL/6J mice (Sankyo Labo Service Corporation, Inc., Hamamatsu, Japan) were housed in individual cages and maintained on a 12-h light/dark cycle with free access to food and water. The body weight of the mice was 22-27 g. Each mouse was anesthetized by inhaled isoflurane (Mylan Pharmaceutical Co., Ltd., Osaka, Japan), after which their dorsal hair was removed by shaving with an electric razor, followed by application of a depilatory agent. Two excisional wounds were pro- duced, on either side of the midline, with a 6-mm biopsy punch (Kai Industries Co., Ltd., Gifu Pref., Japan). A circular silicone splint with an inner diameter of $10 \mathrm{~mm}$ and an outer diameter of $15 \mathrm{~mm}$ was then adhered to the normal skin around the wound, to minimize contraction. Six to eight stitches were placed to keep the splint in place $^{21}$. This excisional splint model is often used to study wound healing.

PDT was started with topical application of a secondgeneration photosensitizer, methyl 5-aminolevulinate hydrochloride (MAL; Tokyo Medical Industrial, Tokyo, Japan), onto the right-side wound. A cotton pad was then placed over the right-side wound to keep the MAL solution in place. The wound was covered with tin foil to prevent light exposure. After 1 to 3 hours of incubation, the tin foil and cotton pad were removed and the whole body, except for the right-side PDT-treated wound, was covered with tin foil. The right-side wound was then irradiated with red light (Photo Therapeutics Ltd., Cheshire, UK; wavelength, $633 \mathrm{~nm}$; power, $1 \mathrm{~kW}$ ). The wholebody coverage with tin foil served to prevent extra illumination. The left-side wound was not treated with MAL or red light. After PDT, the wounds were covered with AIRWALL (Skinix Kyowa Ltd., Osaka, Japan) and bandaged for protection. On Day 3, the dressings were changed and the wounds were photographed with a digital camera. This process was repeated on Days 5, 7, 10, 12, and 14. All experiments were approved by the Ethics Committee of Nippon Medical School and were performed in accordance with the institutional and national guidelines for the care and use of laboratory animals.

On Day 14, the mice were euthanized with a lethal dose of anesthesia. The wounds were excised and fixed in $10 \%$ formalin for $72 \mathrm{~h}$ before being embedded in paraffin. The wound blocks were then sectioned into $4 \mu \mathrm{m}-$ thick slices and the slices were deparaffinized. The sections were subjected to histological analysis with hematoxylin-eosin (HE) and Masson trichrome (MTC) staining. The sections were also subjected to immunohistochemical (IHC) analysis to determine expressions of CD31, collagen I, and collagen III. For this, antigen retrieval was performed in citrate buffer (Muto Pure Chemicals Co., Ltd., Tokyo, Japan; $\mathrm{pH}, 6$ ) at $98^{\circ} \mathrm{C}$ for 20 min, with pressure. To eliminate endogenous peroxidase activity and nonspecific staining, sections intended for CD31, collagen I, and collagen III analysis were respectively blocked for 10, 15, and $30 \mathrm{~min}$ in $0.3 \%$ hydrogen peroxide (Wako Pure Chemical Industries, Ltd., Osaka, 
Japan). The sections were then incubated for $1 \mathrm{~h}$ with primary antibodies specific for CD31 $(1 \mu \mathrm{g} / \mathrm{mL}$, Abcam; Cambridge, MA, USA), collagen I $(5 \mu \mathrm{g} / \mathrm{mL}$, Abcam; Cambridge, MA, USA), or collagen III $(1 \mu \mathrm{g} / \mathrm{mL}$, Abcam; Cambridge, MA, USA). Vectastain ABC rabbit IgG Kit (Funakoshi Co., Ltd., Tokyo, Japan) served as the secondary antibody.

Histological and IHC sections were examined under a microscope (Olympus, Tokyo, Japan), and images were captured with cellSens Standard software (Olympus, Tokyo, Japan). Photographs of the gross morphology were used to determine the effect of PDT on reepithelialization on Days 3, 5, 7, 10, 12, and 14. For this, the wound area was measured and expressed relative to the total wound area on Day 0 , which was defined as $0 \%$ re-epithelialization. A score of $100 \%$ re-epithelialization thus indicated complete wound epithelialization. MTCstained histology images were used to quantify dermal thickness and density of collagen fibers in the wound on Day 14. The IHC images were used to measure the numbers of $\mathrm{CD} 1^{+}$blood vessels and density of collagen I and collagen III.

All quantifications were performed by using Image J 1.52a (Wayne Rasband, National Institutes of Health, USA). All data were expressed as mean \pm SD and were analyzed by using SPSS Statistics 23.0 (IBM Co., Armonk, NY, USA). The PDT-treated and untreated wounds were compared in relation to quantitative variables by using the paired $t$-test. The mutual effects were compared by two-way analysis of variances (ANOVA). A $P$ value of $<0.05$ was considered to indicate statistical significance.

\section{Results}

To identify PDT parameters with the greatest effect in the acute wound healing murine model, we performed a pilot experiment in which the mice were subjected to PDT regimens that varied in incubation time $(1,2$, or $3 \mathrm{~h}$ ), MAL concentration $(5 \%, 10 \%$, or $20 \%)$, and irradiation energy $\left(60,80\right.$, or $\left.100 \mathrm{~mW} / \mathrm{cm}^{2}\right)$. The primary outcome was degree of re-epithelialization, which was expressed as change in wound area on Day 14 relative to wound area on Day 0. As compared with the negative control, wounds treated with light only $\left(60,80\right.$, or $\left.100 \mathrm{~mW} / \mathrm{cm}^{2}\right)$ exhibited accelerated healing, while wounds treated with MAL only $(5 \%, 10 \%$, or $20 \%$ ) showed no difference in wound healing (data not shown). PDT (MAL + light) had no effect on wound healing, unless the incubation time was $3 \mathrm{~h}$. Specifically, all PDT regimens involving a 3-h incubation time had an unexpected effect on wound healing, namely, delayed re-epithelialization on Day 14 . Only MAL + light-treated wounds exhibited decelerated wound healing. The PDT parameters with the greatest effect on Day 14 re-epithelialization were 20\% MAL, a 3-h incubation, and a light energy of $100 \mathrm{~mW} / \mathrm{cm}^{2}$ (data not shown).

To confirm this result and determine why PDT delayed healing of excisional splinted wounds in our model of acute wound healing, we used the most severe PDT parameters, as identified in the pilot study, to repeat the experiment in five mice. Thus, the right-side excisional wound was treated with $20 \% \mathrm{MAL}$, incubated for $3 \mathrm{~h}$, and irradiated with $100 \mathrm{~mW} / \mathrm{cm}^{2}$ light. The left-side wound was left untreated. Morphological photos taken on Day $0,3,5,7,10,12$, and 14 were assessed for reepithelialization. During the first week, the wound area gradually decreased on both sides; no difference was seen between the two sides. However, during the second week, the PDT-treated wound started exhibiting delayed re-epithelialization (Fig. 1).

We then quantified the degree of re-epithelialization over the 14-day observation period by measuring wound area with Image J 1.52a. Thus, wound area on Day 0 was considered to indicate $0 \%$ re-epithelialization, and $100 \%$ re-epithelialization indicated full re-epithelialization. PDT-treated wounds started to exhibit a delay in reepithelialization on Day 3. This difference progressively increased and was significant on Days 10, 12, and 14 ( $p=$ $0.016, p<0.001$, and $p=0.002$, respectively; Fig. 2 ). Thus, on Day 7 , the average areas of PDT-treated and untreated wounds were $42.05 \pm 13.20 \%$ and $55.09 \pm 6.24 \%$, respectively ( $p=0.081$; Fig. 2). Later, on Day 12 , the untreated wounds exhibited complete epithelialization, whereas approximately one quarter of the PDT-treated wounds remained unepithelialized $(0.27 \pm 0.46 \%$ vs. $24.59 \pm 3.59 \%, p<$ 0.001 ; Fig. 2). The areas of PDT-treated wounds and untreated wounds differed at all different time points (ANOVA, $p=0.001$ ).

To determine why PDT delayed re-epithelialization, we harvested wounds on Day 14 and subjected wound sections to histological and IHC analysis. HE staining showed that untreated wounds had a continuous epithelial lining. Thus, the Day 14 untreated wound had undergone complete keratinization and now had an intact epidermis. By contrast, PDT-treated wounds partially lacked epithelium in the wound bed. MTC staining also showed that, as compared with the untreated wounds, PDTtreated wounds had a thicker dermis ( $p=0.039$; Fig. $3 \mathbf{b}$ ) and more collagen fibers ( $p=0.009$; Fig. $3 c$ ) and inflamma- 


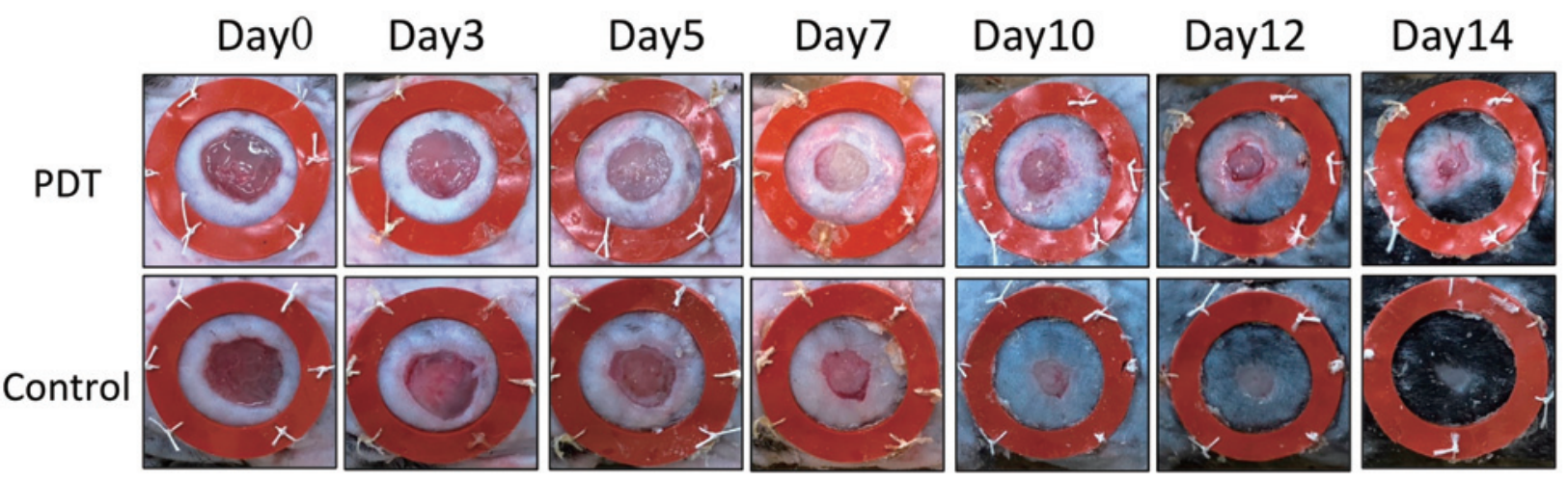

Fig. 1 Images showing gross morphological changes in wounds at different time points after photodynamic therapy (PDT). PDT comprised topical application of methyl 5-aminolevulinate hydrochloride (MAL) for $3 \mathrm{~h}$, followed by irradiation with red light. The wound on the left side (control) was not treated with MAL or red light. The PDT-treated and untreated wounds did not differ in appearance during the first week. However, during the second week, PDT-treated wounds exhibited delayed re-epithelialization (ie, a larger wound area) and greater erythema. Representative images of wounds in 5 mice are shown.

\section{Wound area}

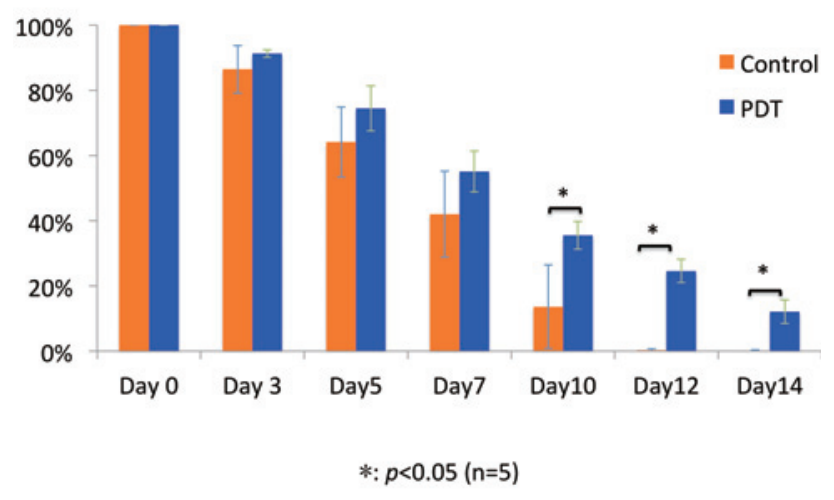

Fig. 2 Change in wound area in relation to baseline (Day 0) at different time points after photodynamic therapy (PDT). PDT delayed re-epithelialization, and wound area was significantly larger for PDTtreated wounds on Days 10, 12, and $14(p<0.05)$. Wound area on Day 0 was defined as $100 \%$. Complete epithelialization was expressed as $0 \%$. The data are expressed as mean $\pm \mathrm{SD}$, and differences were compared with the paired $t$-test ( $n=5$ mice from one independent experiment).

tory cells (Fig. 3a; data not shown). None of the wounds exhibited any evidence of necrosis, edema, or abscess. Thus, the histology results in Figure $3 \mathbf{a} \sim_{\mathbf{c}}$ were consistent with the gross morphological changes on Day 14, shown in Figure 1.

IHC analyses showed that, as compared with the untreated wounds, PDT-treated wounds had significantly fewer $\mathrm{CD} 1^{+}$blood vessels ( $p=0.001$; Fig. 4) and greater collagen III density on Day 14 ( $p=0.021$; Fig. 3d). However, Day 14 collagen I density did not differ between the treated and untreated wounds ( $p=0.692 ;$ Fig. 3c).

\section{Discussion}

PDT is a photochemical therapy comprising a photosensitizer and light. In our study, wounds treated only with light exhibited accelerated healing, which was consistent with previous findings ${ }^{22}$. A possible reason for this finding is that red-light irradiation stimulates proliferation of skin epidermal cells, vascular endothelial cells, and fibrous tissue ${ }^{23}$. Treatment with MAL only had no effect on wound healing in the present study. MAL is a photosensitizer and will not work without light irradiation. Therefore, PDT (MAL + light) seemed to have some effect on wound healing.

However, PDT hampered secondary intention wound healing by delaying re-epithelialization. This effect was associated with the presence of a thicker dermis, which contained fewer blood vessels, greater inflammatory infiltrate, and more collagen fibers and collagen III (but not collagen I). These findings are consistent with those of a study by Mill et al, which showed that PDT treatment of excisional wounds in healthy humans delayed reepithelialization and that this effect was associated with greater inflammation ${ }^{5}$.

The excisional splinted wound model in mice is a model of wound healing. While the acute skin wounds of healthy individuals heal quickly and in an orderly manner, healing of chronic wounds such as diabetic ulcers, pressure ulcers, venous ulcers, and arterialinsufficiency ulcers is disordered and characterized by a prolonged inflammatory phase $e^{24,25}$. Several studies have shown that serial PDT treatments over 3 weeks signifi- 


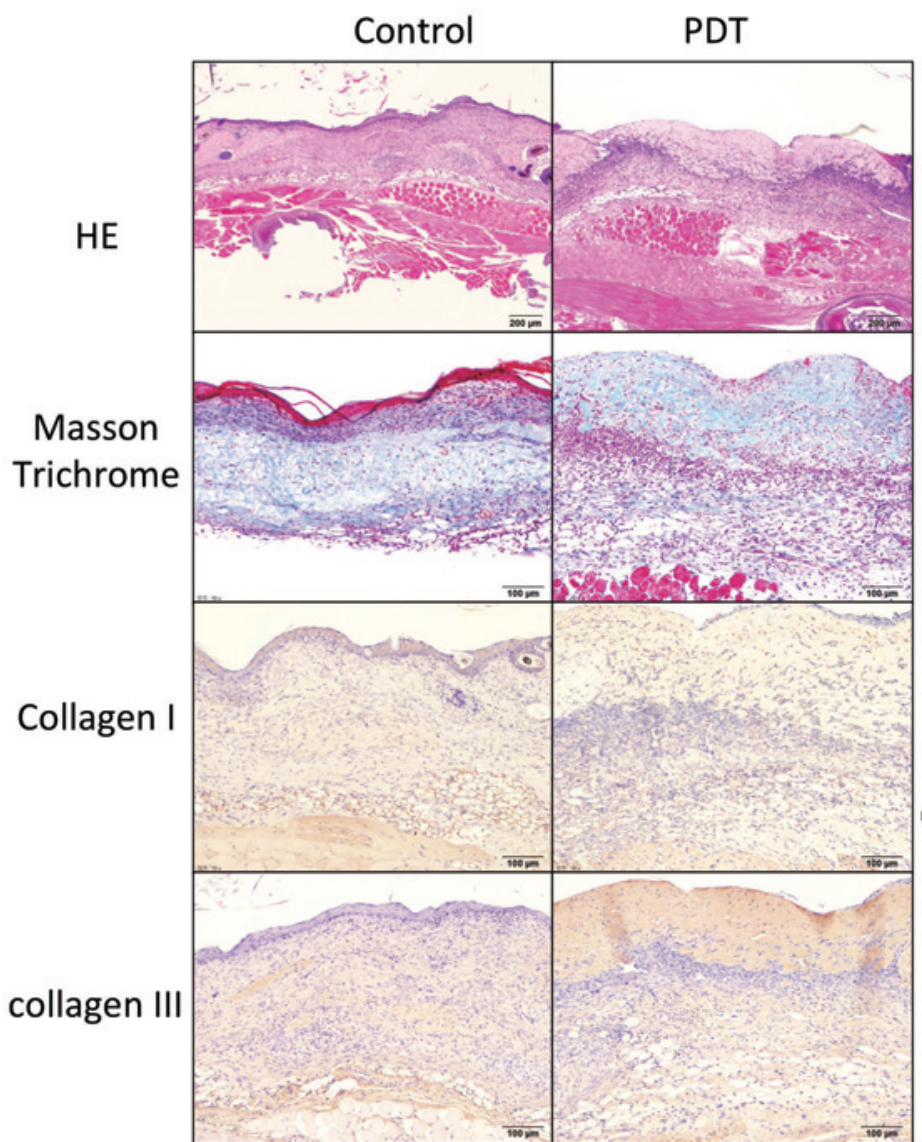

(a)

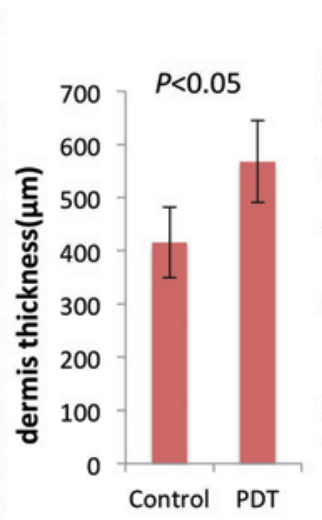

(b)

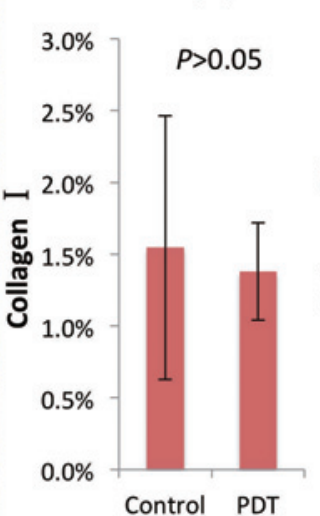

(d)

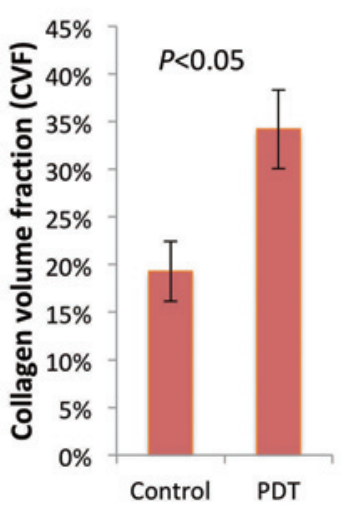

(c)

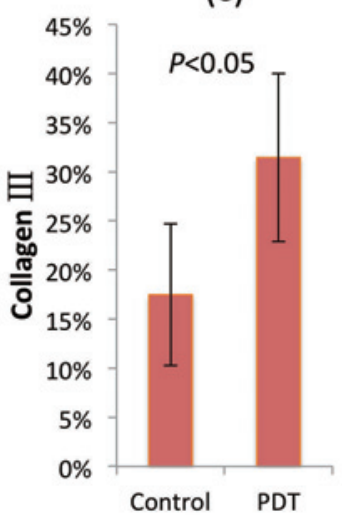

(e)

Fig. 3 Histological images of murine wounds (a) and average values for wound healing variables (b-e) on Day 14 after photodynamic therapy (PDT). (a) Images of the control and PDT-treated wounds after hematoxylin-eosin staining (magnification $\times 40$ ), Masson trichrome (MTC) staining $(\times 100)$, and immunohistochemical (IHC) staining for collagen I $(\times 100)$ and collagen III $(\times 100)$. Representative images of the wounds of 5 mice are shown. $(b$ and $c)$ The average dermal thickness of the wounds (b) and density of collagen fibers (c) were calculated on the basis of MTC-stained images. ( $d$ and e) The average densities of collagen I (d) and collagen III (e) were calculated on the basis of IHC images. There were statistically significant differences $(p<0.05$, paired $t$-test) between treated and untreated wounds in b, c, and e but not in $\mathrm{d}$. The data in b-e are expressed as mean \pm SD ( $n=5$ mice from one independent experiment).

cantly improved closure of chronic venous ulcers ${ }^{17}$ and diabetic ulcers ${ }^{18}$. Our finding that PDT delayed the closure of wounds in the present animal model appears to contradict these previous findings.

There are two, non-mutually exclusive, possibilities that explain this apparent discrepancy. First, infection is a crucial factor in poor wound healing, and PDT has a powerful antimicrobial effect ${ }^{192,2,26,27}$. Isolated Staphylococcus aureus from infected human burn wounds is highly sensitive to PDT $^{19}$ : a single PDT session killed $98 \%$ of human burn-derived S. aureus bacteria in murine burn wounds ${ }^{20}$. Thus, PDT may promote chronic wound healing in humans by eliminating infections that delay wound healing. Second, a study of PDT-treated excisional wounds in healthy humans showed that although PDT delayed reepithelialization during the inflammatory phase, it was associated with a significantly smaller wound area during the remodeling phase at Week 3 and better cosmetic outcomes and more ordered dermal structure at 9 months $s^{5}$. Thus, if we had extended our observation period beyond 14 days, we might have found that PDT ultimately had beneficial effects on wound healing in our murine model of secondary wound healing.

Cutaneous wound healing is a complex, dynamic process that is mediated by interactions between multiple cell types, cytokines, and chemokines. The wound healing processes that PDT initially hampered in the present and past studies ${ }^{5}$ are unclear. However, some may be related to fibroblasts, a key cell in normal wound healing. By secreting and depositing elastin and collagens into a dermal defect, fibroblasts act to restore tissue integrity after wounding. This process changes over time: the initially 


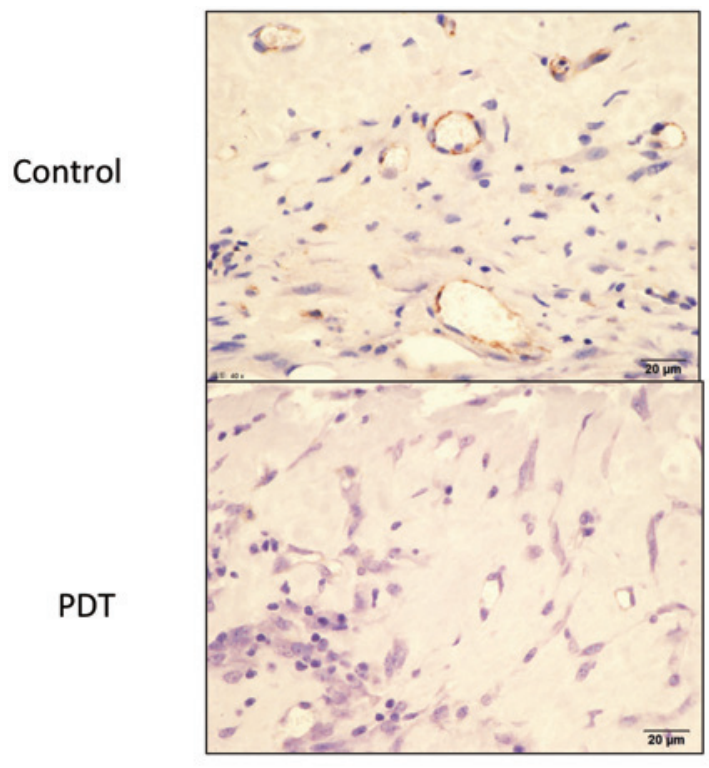

(a)

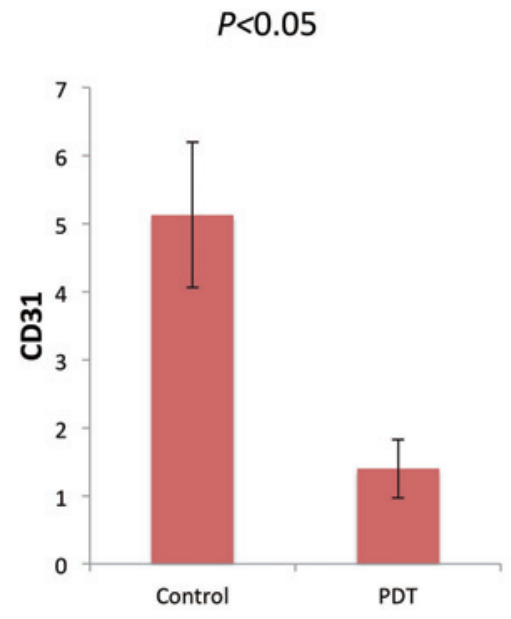

(b)

Fig. 4 Images of immunohistochemical staining for CD31 $(\times 400)$ in murine wounds (a), and average number of CD31+ blood vessels (b) on Day 14 after photodynamic therapy. The data are expressed as mean $\pm \mathrm{SD}$ ( $n=5$ mice from one independent experiment). There was a significant difference ( $p<0.05, t$-test) between treated and untreated wounds in $\mathrm{b}$.

high level of collagen III production is overtaken by collagen I production. Eventually, collagen fibrils assume a more orderly appearance. These changes result in dermal remodeling and eventual restoration of dermal strength. In our study, PDT appeared to delay the start of this remodeling phase, as it was associated with significantly more collagen III in the wound area on Day 14.

PDT may also interfere with early angiogenesis. Marked collagen deposition, which starts soon after wound healing, results in the formation of granulation tissue. As this tissue begins to form, new blood vessels grow into it to feed it with nutrients and oxygen. These vessels allow the influx of inflammatory cells, which are important in proper wound healing ${ }^{28}$. A key cytokine in these events, TGF- $\beta$, includes TGF- $\beta 1$ and TGF- $\beta 2$, which promote expression of collagen I, collagen III, and the pro-angiogenic cytokine vascular endothelial growth factor $(\mathrm{VEGF})^{29}$. A third isotype, TGF- $\beta 3$, may play an important downregulatory role in wound healing by suppressing the inflammatory process, which has been implicated in the development of small, unnoticeable scars ${ }^{30,31}$. In addition to promoting angiogenesis, VEGF stimulates re-epithelialization and helps resolve the inflammatory phase of wound healing ${ }^{32,33}$. Thus, TGF- $\beta 1$ and TGF- $\beta 2$ ultimately promote re-epithelialization, in part by driving angiogenesis, while TGF- $\beta 3$ may modulate this activity. In our study, PDT was associated with a decrease in the number of $\mathrm{CD} 31^{+}$blood vessels on Day 14. Thus, PDT may have delayed re-epithelialization in our model of secondary intention wound healing by suppressing TGF$\beta 1$-driven angiogenesis during the proliferative phase of wound healing. This possibility is supported by the study of Mills et al, which found that TGF- $\beta 1$ levels were lower on Day 7 in PDF-treated excisional wounds than in untreated wounds.

The remodeling phase is the last step of wound healing and involves the disappearance of existing cells, formation of new cells, and production of an ordered collagen matrix. Any disturbance that occurs during this phase can lead to chronic or excessive wound healing ${ }^{34}$. Matrix metalloproteinases (MMP) are important in reepithelialization and matrix remodeling. Mills et al reported that, during the remodeling phase at Week 3, MMP-1 and MMP-9 levels were significantly higher in PDT-treated wounds of healthy subjects than in untreated wounds. This may explain their finding that PDT was associated with significant improvement in the architecture of the deposited matrix at 9 months ${ }^{5}$.

In conclusion, PDT delayed acute wound healing in a murine model of secondary intention wound healing. During the second week, re-epithelialization progressed significantly more slowly in PDT-treated wounds than in untreated wounds. This effect may be mediated by the suppressive effect of PDT on collagen production pat- 
terns and angiogenesis. However, previous findings suggest that PDT has positive effects on excisional wound healing at later time points ${ }^{5}$. Thus, further studies with longer observation times and larger sample sizes are needed. Clarification of the effect of PDT on production of key wound-healing cytokines such as TGF- $\beta 3$ and VEGF in our model would be of particular interest.

Conflict of Interest: The authors have no conflicts of interest to declare and all authors have signed the declaration of copyright transfer.

\section{References}

1. Kiya K, Kubo T. Neurovascular interactions in skin wound healing. Neurochem Int. 2019;125:144-50.

2. Pazyar N, Yaghoobi R, Rafiee E, Mehrabian A, Feily A. Skin wound healing and phytomedicine: a review. Skin Pharmacol Physiol. 2014;27:303-10.

3. Kasuya A, Tokura Y. Attempts to accelerate wound healing. J Dermatol Sci. 2014;76:169-72.

4. Posnett J, Gottrup F, Lundgren H, Saal G. The resource impact of wounds on health-care providers in Europe. J Wound Care. 2009;18:154-61.

5. Mills SJ, Farrar MD, Ashcroft GS, Griffiths CE, Hardman MJ, Rhodes LE. Topical photodynamic therapy following excisional wounding of human skin increases production of transforming growth factor-beta3 and matrix metalloproteinases 1 and 9, with associated improvement in dermal matrix organization. Br J Dermatol. 2014;171:55-62.

6. Katayama B, Ozawa T, Morimoto K, et al. Enhanced sterilization and healing of cutaneous pseudomonas infection using 5-aminolevulinic acid as a photosensitizer with 410nm LED light. J Dermatol Sci. 2018;90:323-31.

7. Wang T, Li X, Fan L, et al. Negative pressure wound therapy promoted wound healing by suppressing inflammation via down-regulating MAPK-JNK signaling pathway in diabetic foot patients. Diabetes Res Clin Pract. 2019; 150:81-9.

8. Fearmonti RM. Efficacy of epidermal skin grafts over complex, chronic wounds in patients with multiple comorbidities. Wounds. 2016;28:226-32.

9. Duque APD, Pinto NDC, Mendes RD, et al. In vivo wound healing activity of gels containing Cecropia pachystachya leaves. J Pharm Pharmacol. 2016;68:128-38.

10. Ozog DM, Rkein AM, Fabi SG, et al. Photodynamic therapy: A clinical consensus guide. Dermatol Surg. 2016;42 (7):804-27.

11. Kou J, Dou D, Yang L. Porphyrin photosensitizers in photodynamic therapy and its applications. Oncotarget. 2017; 8:81591-603.

12. Jerjes W, Hamdoon Z, Hopper C. Photodynamic therapy in the management of basal cell carcinoma: Retrospective evaluation of outcome. Photodiagnosis Photodyn Ther. 2017;19:22-7.

13. Victoria-Martinez AM, Martinez-Leborans L, OrtizSalvador JM, Perez-Ferriols A. Treatment of bowen disease with photodynamic therapy and the advantages of sequential topical imiquimod. Actas Dermo-Sifilogr. 2017; 108:E9-14.

14. Jerjes W, Hamdoon Z, Abdulkareem AA, Hopper C. Photodynamic therapy in the management of actinic kerato- sis: Retrospective evaluation of outcome. Photodiagnosis Photodyn Ther. 2017;17:200-4.

15. Boen M, Brownell J, Patel P, Tsoukas MM. The role of photodynamic therapy in acne: An evidence-based review. Am J Clin Dermatol. 2017;18:311-21.

16. Sun Y, Chen L, Zhang Y, Gao X, Wu Y, Chen H. Topical photodynamic therapy with 5-aminolevulinic acid in Chinese patients with Rosacea. J Cosmet Laser Ther. 2019;21 (4):196-200.

17. Grandi V, Bacci S, Corsi A, et al. ALA-PDT exerts beneficial effects on chronic venous ulcers by inducing changes in inflammatory microenvironment, especially through increased TGF-beta release: A pilot clinical and translational study. Photodiagn Photodyn. 2018;21:252-6.

18. Carrinho PM, Andreani DIK, Morete VD, Iseri S, Navarro RS, Villaverde AB. A study on the macroscopic morphometry of the lesion area on diabetic ulcers in humans treated with photodynamic therapy using two methods of measurement. Photomed Laser Surg. 2018;36: $44-50$.

19. Mahmoudi H, Pourhajibagher M, Alikhani MY, Bahador A. The effect of antimicrobial photodynamic therapy on the expression of biofilm associated genes in Staphylococcus aureus strains isolated from wound infections in burn patients. Photodiagnosis Photodyn Ther. 2019;25:406-13.

20. Lambrechts SAG, Demidova TN, Aalders MCG, Hasan T, Hamblin MR. Photodynamic therapy for Staphylococcus aureus infected burn wounds in mice. Photoch Photobio Sci. 2005;4:503-9.

21. Dunn L, Prosser HC, Tan JT, Vanags LZ, Ng MK, Bursill CA. Murine model of wound healing. J Vis Exp. 2013; (75):e50265.

22. Wu X, Alberico S, Saidu E, et al. Organic light emitting diode improves diabetic cutaneous wound healing in rats. Wound Repair Regen. 2015;23:104-14.

23. Li Y, Zhang J, Xu Y, et al. Effects of $630 \mathrm{~nm}$ Red and 460 $\mathrm{nm}$ blue light emitting diode irradiation on healing of the skin wound in Japanese big-ear white rabbit. Acta Academiae Medicinae Sinicae. 2017 Jun 20;39:301-6.

24. Singla R, Soni S, Patial V, et al. Cytocompatible antimicrobial dressings of syzygium cumini cellulose nanocrystals decorated with silver nanoparticles accelerate acute and diabetic wound healing. Sci Rep. 2017;7:10457.

25. Nicholas MN, Yeung J. Current status and future of skin substitutes for chronic wound healing. J Cutan Med Surg. 2017;21:23-30.

26. Sahu K, Sharma M, Dube A, Gupta PK. Topical antimicrobial photodynamic therapy improves angiogenesis in wounds of diabetic mice. Lasers Med Sci. 2015;30:1923-9.

27. Lei X, Liu B, Huang Z, Wu J. A clinical study of photodynamic therapy for chronic skin ulcers in lower limbs infected with Pseudomonas aeruginosa. Arch Dermatol Res. 2015;307:49-55.

28. Schultz GS, Wysocki A. Interactions between extracellular matrix and growth factors in wound healing. Wound Repair Regen. 2009;17:153-62.

29. Barrientos S, Stojadinovic O, Golinko MS, Brem H, TomicCanic M. Growth factors and cytokines in wound healing. Wound Repair Regen. 2008;16:585-601.

30. Chang Z, Kishimoto Y, Hasan A, Welham NV. TGF-beta3 modulates the inflammatory environment and reduces scar formation following vocal fold mucosal injury in rats. Dis Model Mech. 2014;7:83-91.

31. Lichtman MK, Otero-Vinas $M$, Falanga V. Transforming growth factor beta (TGF-beta) isoforms in wound healing and fibrosis. Wound Repair Regen. 2016;24:215-22. 
32. Bienert $M$, Hoss $M$, Bartneck $M$, et al. Growth factor(Received, June 14, 2019) functionalized silk membranes support wound healing in (Accepted, October 29, 2019)

33. Johnson KE, Wilgus TA. Vascular endothelial growth factor and angiogenesis in the regulation of cutaneous wound repair. Adv Wound Care (New Rochelle). 2014;3: 647-61.

34. Tsai HW, Wang PH, Tsui KH. Mesenchymal stem cell in wound healing and regeneration. J Chin Med Assoc. 2018;81:223-4.

Journal of Nippon Medical School has adopted the Creative Commons Attribution-NonCommercial-NoDerivatives 4.0 International License (https://creativecommons.org/licenses/by-nc-nd/4.0/) for this article. The Medical Association of Nippon Medical School remains the copyright holder of all articles. Anyone may download, reuse, copy, reprint, or distribute articles for non-profit purposes under this license, on condition that the authors of the articles are properly credited. 